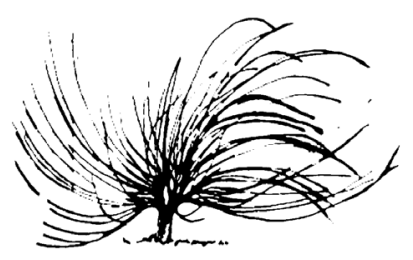

\title{
Teorías de aprendizaje y su relación en la educación ambiental costarricense
}

\author{
Luis Diego Mesén Mora ${ }^{1}$ \\ Universidad Nacional \\ Costa Rica \\ luis.mesen.mora@una.ac.cr
}

\begin{abstract}
Resumen
Durante muchos años se ha buscado optimizar la experiencia educativa con el fin de mejorar la transposición pedagógica en el proceso de enseñanza y aprendizaje. Esto llevó al desarrollo de diversas teorías de aprendizaje, entre ellas el conductismo, cognitivismo, constructivismo, socioconstructivismo, conectivismo, entre otras. Cada una de ellas, basada en su epistemología, fomenta el desarrollo del proceso de enseñanza y aprendizaje de una manera especializada. El objetivo del presente análisis se fundamenta en una descripción de las teorías de aprendizaje mencionadas y cómo se relacionan con la educación ambiental en el sistema educativo costarricense. Mediante el estudio bibliográfico y el análisis de algunas actividades educativas socioambientales, se relacionó el uso de estas teorías, con el desarrollo de diversas habilidades enfocadas en promover la importancia de los recursos naturales y la conservación de estos para su uso racional por las futuras generaciones.
\end{abstract}

Palabras clave: Educación ambiental, teorías de aprendizaje, manejo de recursos naturales.

\section{(c) (i) (9)}

Recibido: 6 de junio de 2018. Aprobado: 10 de abril de 2019.

http://dx.doi.org/10.15359/rep.14-1.8

1 Licenciado en Química Industrial, candidato Maestría en Educación con énfasis en Pedagogía Universitaria, Universidad Nacional, Costa Rica 


\begin{abstract}
For many years, improving the educational experience to have better pedagogical transposition in teaching and learning processes has been sought. This led to the development of various theories of learning, including behaviorism, cognitivism, constructivism, socioconstructivism and conectivism. Each of them, based on its epistemology, fosters the development of teaching and learning process in a specialized manner. The objective of this analysis is centered on a description of learning theories and how they relate to environmental education in the Costa Rican Educational system. Through bibliographic study and the analysis of some socio-environmental educational activities, the use of these theories was related to the development of diverse skills focused on promoting the importance of natural resources and the conservation of these for their rational use by future generations.
\end{abstract}

Keywords: environmental education, learning theories, natural resource management.

\title{
Introducción
}

Uno de los principales retos de la educación actual es el desarrollo de la educación ambiental y su relación con el desarrollo sostenible. Es importante acudir a los procesos de enseñanza y aprendizaje para inculcar en las futuras generaciones la necesidad de manejar y gestionar de manera adecuada los recursos naturales. De esta manera, la educación es la principal herramienta para el desarrollo sociocultural en busca de la preservación de los recursos naturales de una forma sostenible. El conocimiento básico de diversas teorías de aprendizaje y su aplicación en el desarrollo de la educación ambiental permite conocer diferentes maneras de mediar sobre la preservación del medio ambiente con jóvenes costarricenses, desde los primeros años de sus procesos educativos.

A través del tiempo, se han desarrollado diversas teorías de aprendizaje, las cuales nacen como "estrategias que poseen los docentes para desarrollar la didáctica y mejorar los procesos de enseñanza y aprendizaje" (Villalobos, 2002, p. 78). El personal docente debe tener un papel 
preponderante como guía de sus estudiantes, de esta manera, puede mediar en el desarrollo de sus diferentes habilidades, de la mano con un aprendizaje significativo (Sierra, Morales y Barrios, 2014). Esto confirma la importancia de una base de conocimiento epistemológico por parte de los grupos docentes, lo cual permite lograr una transposición pedagógica eficiente, diversificar la educación y obtener una mejor comprensión por parte de su alumnado. Cada uno de los actores sociales propios del proceso de enseñanza y aprendizaje posee diferentes características y aptitudes, por lo que la identificación de estas le permitirá al personal docente utilizar y diseñar los recursos pertinentes para que cada estudiante maximice el aprendizaje de los conceptos y habilidades meta (Torrentera, 2015).

El uso adecuado de los recursos naturales, así como propiciar la conservación biológica, es uno de los principales retos que enfrentan las nuevas generaciones. De ahí la importancia de relacionar, de manera adecuada, la educación y el manejo de estos. El desarrollo cultural y un estilo de vida bueno para las futuras generaciones está ligado directamente a los esfuerzos que las generaciones actuales realicen, lo que confirma la importancia de la educación ambiental integrada en el proceso de enseñanza y aprendizaje. Es preponderante crear conciencia sobre cómo utilizar los recursos naturales, pero de manera sostenible y la principal herramienta para lograr esta tarea radica en los procesos de enseñanza y aprendizaje con un énfasis en la educación ambiental. Velar por el uso y regeneración de recursos, el cuido de especies en peligro, la conservación del agua, del suelo, del aire, así como de los elementos biológicos y bioquímicos que forman parte de nuestro diario vivir son parte de las responsabilidades sociales que podemos comprender y afrontar con una educación básica integradora. Como seres sociales, debemos aprender a desarrollarnos en equilibrio desde puntos de vista económico, social y ambiental; de esta manera se puede lograr un desarrollo integral que permita que nuevas generaciones tengan acceso a los recursos actuales (Novo y Zaragoza, 2006).

Este desarrollo solo se puede lograr con una estructura educativa que tenga una base amplia de conceptos ambientales y de conservación de recursos desde las primeras etapas del proceso educativo. Es necesario un cambio en el enfoque del desarrollo actual más consumista hacía un desarrollo sostenible que permita que las generaciones futuras tengan acceso a los recursos naturales de calidad (Picazzo, Gutiérrez, Infante y Cantú, 2011). 
Existen diversas metodologías que pueden ser utilizadas por docentes en sus aulas, las cuales se asocian a las diversas teorías de aprendizaje desarrolladas. Por ende, la comprensión y el análisis de estas se presentan como herramientas fundamentales para lograr que el estudiantado cumpla los objetivos propuestos a nivel educativo y crear conciencia sobre la necesidad del desarrollo sostenible para la ejecución y mejoramiento de la conservación biológica a través de los años.

Los recursos naturales son determinantes para el desarrollo país, por ende, la necesidad de una educación ambiental basada en un fundamento epistemológico claro y definido, que permita a docentes elaborar los instrumentos y actividades idóneas para lograr las metas educativas. El presente análisis pretende relacionar algunas nociones básicas de las teorías de aprendizaje tradicionales y modernas con diversos elementos que son aplicados actualmente en los diferentes niveles educativos. De esta manera, exhortar a los diversos actores del proceso de enseñanza y aprendizaje a profundizar e innovar acerca de la necesidad de un desarrollo integral que permita una mejora progresiva en la educación ambiental con miras a un desarrollo sostenible.

\section{La transposición pedagógica}

El principal fin de los procesos educativos es mejorar el aprendizaje significativo de los sujetos actores sociales (Francisco y González, 2017). De esta manera y como motor de cambio, se puede lograr una evolución social que esté de la mano con el desarrollo del país. El personal docente tiene como una de sus principales metas que el estudiantado logre comprender, de manera integral, los conceptos y temáticas estudiadas en clase. Este entendimiento está ligado al uso correcto de las teorías de aprendizaje, lo que permite la selección de las actividades de mediación correctas, las técnicas de clase y demás elementos que fomenten un adecuado crecimiento educativo.

Este mecanismo por el cual "los contenidos son un conjunto de productos culturales seleccionados para ser aprendidos por los alumnos, con alguna intención y en contextos específicos" (Bravo, 2005, p. 34) se denomina transposición pedagógica, la cual tiene como principal objetivo la unión entre los saberes previos del estudiantado con los saberes creados durante el proceso de enseñanza y aprendizaje. Esta transposición es el puente por el cual el estudiantado comprende, 
asimila, domina, analiza y aplica los contenidos pertenecientes al sistema educativo para ser utilizados en su vida profesional, de manera integral, como seres sociales activos.

\section{Una mirada a las teorías de aprendizaje tradicionales}

El desarrollo de las diversas teorías de aprendizaje nació como una necesidad de mejorar los procesos de enseñanza y aprendizaje, basados en entender cómo el estudiantado aprende de manera más efectiva (Alonso, 2008). A través de los años, se desarrollaron tres teorías clásicas: el conductismo, el cognitivismo y el constructivismo (Cejudo y Almenara, 2015). Cada una, presenta ventajas y desventajas, lo que ha llevado a no tomar ninguna teoría como absoluta, sino a buscar una especialización y desarrollo en una teoría especifica según las características docentes y su desarrollo académico (Pérez, Miguelena y Diallo, 2016). La comprensión y aplicación de cada teoría habilita al personal docente para alcanzar una mejor transposición pedagógica con el estudiantado, de acuerdo con sus características y capacidades, para generar un mejor ambiente de aprendizaje en el proceso.

\section{Conductismo}

El conductismo se basa en la repetición de diferentes acciones para lograr el aprendizaje requerido en respuesta a las mismas acciones (Torrenteras, 2015). El personal docente se caracteriza por dirigir las actividades desarrolladas, en donde, por medio de refuerzos y castigos, se pretende que el alumnado obtenga las conductas y conceptos requeridos para eliminar lo no deseado (Cejudo y Almenara, 2015).

En esta teoría de aprendizaje, los pensamientos y sentimientos (conocidos como "caja negra") no son relevantes, ya que se consideran propios de cada ser humano sin influir en el proceso de aprendizaje, solo importa lo que está fuera de esa "caja negra" (Rojas, Rojas y Fernández, 2016). Mientras, se consideran pilares del conductismo el grado con el que el alumnado se refuerza para cumplir la tarea asignada y su estado de desarrollo mental y físico (Schunk, 1997).

La epistemología del modelo conductista se basa en transmitir, el modelo se centra en la enseñanza, y la enseñanza se centra en el profesorado (Hilario, 2015). 


\section{Cognitivismo}

El cognitivismo considera la experiencia del estudiantado y su desarrollo cognitivo; a diferencia del conductismo, sí importan los pensamientos y sentimientos (Delgado y Alvarado, 2016). El profesorado debe ser un facilitador para que, por medio del desarrollo de experiencias y recursos novedosos, se fomente el aprendizaje de forma interesante asociado a un conocimiento significativo (Leiva, 2005). Este es un modelo solipsista, el cual se centra en el estudiantado, y el personal docente tiene un papel de facilitador; el estudiantado es el centro y él debe aprender y no el profesorado enseñar (Castañon, 2017). Esta teoría de aprendizaje se deriva de la escuela psicológica alemana que estudia el aprendizaje como un proceso integral donde la comprensión, el análisis y el contexto social forman parte de los elementos necesarios para lograr los objetivos de aprendizaje (Fernández, 2016).

Los cognitivistas utilizan y promueven muchas estrategias de instrucción similar a los conductistas, sin embargo, las razones son diferentes. Mientras un conductista usa la retroalimentación para modificar la conducta en la dirección deseada, un cognitivista la utiliza para guiar y reforzar las conexiones mentales exactas. (Thompson, Simonson y Hargrave, 1992, citados por Martin, Martínez, Martín, Nieto y Núñez, 2017, p. 52)

El cognitivismo tiene una relación intrínseca con las teorías del desarrollo propuestas por Piaget, donde el ser humano presenta cuatro etapas, la primera llamada sensorial-motora, la segunda pre-operaciones y las dos últimas conocidas como operaciones concretas y formales (Pozo, 2016). Es en esta última en donde se crea el conocimiento (en el cognitivismo) a partir de la lógica y de las experiencias recopiladas durante el proceso de enseñanza y aprendizaje.

\section{Constructivismo}

En la teoría constructivista, el aprendizaje se basa en la experiencia de enseñanza del alumnado, donde el conocimiento nuevo se une al ya adquirido, para así generar la creación de lo aprendido mediante nuevas experiencias (Fernández, 2016). Es un proceso continuo, donde el 
estudiantado debe construir su conocimiento basado en las experiencias obtenidas al reflexionar sobre las actividades realizadas en el proceso de aprendizaje (Cejudo y Almenara, 2015).

En este proceso educativo, el personal docente tiene un papel de acompañante y mediador para que el alumnado logre el máximo desarrollo de su aprendizaje (Craveri y Anido, 2014). Epistemológicamente, el proceso de enseñanza y aprendizaje tiene como centro tanto al profesorado como al alumnado y enfatiza en ¿cómo se conoce? y ¿cómo se comunica lo que se conoce?, por ende, el conocimiento es una representación pertinente de la realidad, lo que deriva en la comprensión de cómo se construye el mundo (Labra, 2016).

En este modelo, el profesorado no se considera el dueño absoluto del conocimiento, sino que debe cumplir un rol activo que permita el aprendizaje propio de sus estudiantes de manera continua.

Los constructivistas, al igual que los cognitivistas, ven al estudiante como un elemento activo en el entorno del proceso de aprendizaje, aunque los constructivistas valoran al estudiante como más que un simple procesador activo de información, pues el mismo elabora e interpreta la información que se le ofrece. El estudiante es quien crea el significado, a la vez que los objetivos de aprendizaje no están predeterminados, ni tampoco se prediseña la instrucción. (Martin et al. 2017, p. 54)

Como se mencionó con anterioridad, no existe una teoría dominante, cada docente puede diseñar su clase y su currículo basado en la teoría que le permita abarcar de mejor manera los conceptos y que facilite a sus estudiantes el logro de los objetivos propuestos. (Pérez, Miguelena y Diallo, 2016). A pesar de que estas teorías existen desde hace mucho tiempo, gran cantidad de docentes las siguen desconociendo y no utilizan ningún fundamento teórico o pedagógico para preparar sus clases, lo que representa una realidad un poco desalentadora en algunos casos. Cada teoría tiene aspectos importantes, por tanto, el conocimiento, especialización y dominio de una teoría por parte del personal docente permite lograr un proceso educativo estructurado de la mano con el logro de las metas educativas planteadas. El entendimiento de las teorías tradicionales de aprendizaje permite diseñar metodologías concordantes con el currículo propuesto de la mano de las bases epistemológicas. 


\section{Figura 1.}

\section{Descripción básica de los elementos propios de las teorías de aprendizaje tradicionales}
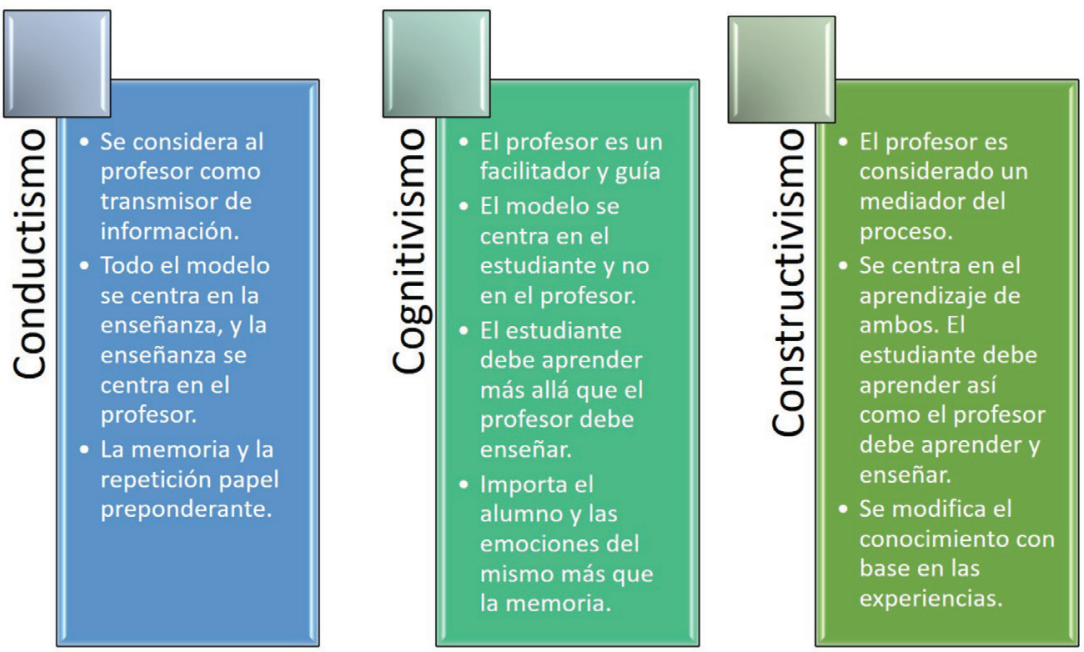

Nota: Elaboración propia.

\section{Teorías de aprendizaje modernas: Socio-constructivismo y conectivismo}

El desarrollo educativo en los últimos años, así como el diseño de nuevas tecnologías ha permitido la creación y evolución de nuevos modelos de aprendizaje, los cuales presentan características diferenciadoras relacionadas con las condiciones socioculturales actuales.

\section{Socio-constructivismo}

Dentro de las teorías modernas, una de ellas es el socio-constructivismo, el cual es una evolución del constructivismo de Vygotsky. En este modelo, la persona cumple un rol activo que busca un aprendizaje gradual. El acto de aprender depende de la relación entre el aprendizaje y la vida. El aprendizaje colectivo es parte del eje central de la enseñanza que potencializará la capacidad de resolver problemas contextualizados mediante la práctica investigativa (Altamirano y Salinas, 2016) En este modelo, la investigación juega un rol primordial, la cual se debe asociar 
con las metodologías y recursos actuales, los cuales permiten procesos más dinámicos con la integración de las tecnologías educativas. De esta manera, se busca promover la construcción de la identidad personal e integrar a las generaciones más jóvenes en un contexto social, que las sitúe de forma competente, participativa, activa y crítica en la sociedad.

\section{Conectivismo}

Otra de las teorías de aprendizaje modernas nace como parte del desarrollo de la revolución tecnológica actual y basada en las herramientas digitales recientes. Esta teoría es denominada conectivismo. Su principal fin se centra en crear una integración didáctica entre nuevos recursos como las tecnologías de la información y la comunicación (TIC), las herramientas de software social para el aula y redes sociales entre otras herramientas con los procesos de enseñanza y aprendizaje (Siemens, 2004).

Esta teoría implica la conexión de las nuevas fuentes tecnológicas con los procesos educativos, lo que conlleva a una profundización y especialización de docentes para lograr una evolución y no quedarse en el uso de las teorías de aprendizaje tradicionalistas

El conectivismo, para sus defensores, supone un estadio superior de los tres modelos anteriores tradicionales (conductismo, cognitivismo y constructivismo), los cuales no consideran el aprendizaje alcanzado fuera de los individuos (o sea, aprendizaje almacenado y manipulado por tecnologías), como tampoco pueden describir cómo ocurre al aprendizaje en colectivo. (Sobrino, 2014, p. 39)

La base del conectivismo se centra en conexiones naturales, las cuales ocurren casi de manera espontánea, sin intención explicita por parte de los individuos que aprenden, incluso, pueden ocurrir sin su control. En este modelo, no cabe hablar de transferencia o construcción del conocimiento (Martin et al. 2017)

El conectivismo define el aprendizaje como un proceso continuo que ocurre en diferentes escenarios como pueden ser comunidades de práctica, redes de personales y en el desempeño de las tareas en el ambiente laboral. Además, sostiene que los modelos tradicionales de aprendizaje fueron desarrollados en una época en la que la tecnología 
no había tenido el impacto en el aprendizaje, tal como ocurre en la actualidad, lo que reafirma la importancia de esta teoría de aprendizaje (Gutiérrez, 2012).

\section{Educación ambiental como herramienta de conciencia ambiental}

Luego de una descripción básica de algunas teorías de aprendizaje, es posible abordar diferentes problemáticas nacionales, como lo es el manejo y la conservación de los recursos naturales. Desde el nuevo paradigma del desarrollo sostenible, es necesario que las futuras generaciones tengan las mismas oportunidades en cuanto al uso de los recursos naturales. Lo anterior nos impulsa a desarrollar la educación a la luz de un componente conservacionista muy marcado. De esta manera, las futuras generaciones podrán acceder a los recursos autóctonos desde una perspectiva de uso más integral.

Debido a la necesidad de fomentar la conservación de los recursos naturales en Costa Rica, tanto las autoridades de las universidades estatales, así como el Ministerio de Educación Pública (MEP) a nivel de secundaria y primaria, han propuesto diversos mecanismo y metodologías para abordar la problemática ambiental. En el caso de las universidades públicas, se ejecutan planes de manejo y conservación en sus estatutos con el fin de fortalecer la conservación de los recursos naturales. Además, las unidades académicas, dentro de sus áreas de especialización, asumen compromisos que permiten mantener un uso racional de los recursos naturales. En el caso del MEP, propone el uso de los ejes transversales y programas que buscan la integración de estudiantes con los esfuerzos conservacionistas (Mata, 2013).

La transversalidad en los planes de estudio incorpora al currículo objetivos educativos específicos que no son parte de las áreas básicas de conocimiento (Aparicio, Rodríguez, Beltrán y Sampedro, 2014). De esta manera, se puede ampliar la formación de estudiantes, no solo conceptualmente, sino en diversos valores y conductas necesarias para un desarrollo integral que involucre todas las asignaturas propias del currículo educativo. En la actualidad, el MEP trabaja la conservación de los recursos naturales mediante el eje transversal "Cultura ambiental para el desarrollo sostenible", el cual busca la innovación de recursos didácticos para promover los valores, aptitudes, destrezas y actitudes necesarias para obtener acciones responsables con el medio ambiente (Mata, 2013). 
Debido a la importancia de la conservación ambiental, los programas de protección y conservación deben estar desarrollados en un marco conceptual y epistemológico definido, relacionado con la teoría de aprendizaje elegida para su implementación. Los programas que busquen instaurar y promover la educación ambiental deben ser dinámicos y novedosos para el estudiantado. De esta manera, la comprensión de los conceptos adquiridos, ya sea directa o transversalmente, será más eficiente y de acuerdo con las necesidades del país.

Es importante que la transposición didáctica no se lleve a cabo por medio de una transmisión pura por parte del profesorado, sino debemos facilitar y mediar en cuanto la construcción y desarrollo del conocimiento. Dentro de un marco epistemológico claro, el cognitivismo y constructivismo son dos importantes herramientas que permiten que el alumnado desarrolle, critique, construya, y no aprenda por una pura repetición, sino por un desarrollo de su entorno con los conceptos e ideas creadas. El socio-constructivismo, a través de la investigación como medio de aprendizaje, y el conectivismo, como modelo aplicado en las tecnologías actuales, son instrumentos novedosas y acordes con las generaciones actuales.

Es importante integrar a todo el alumnado de la institución educativa en los programas destinados al desarrollo socioambiental. De esta manera, se garantiza un crecimiento de generaciones futuras ambientalmente conscientes y con la finalidad de lograr un objetivo en común, como lo es la conservación y la protección de los recursos naturales (Chinchilla, 2017). El trabajo en equipo y como institución es fundamental para el éxito de los programas conservacionistas, por lo que el aprendizaje socio-constructivista y constructivista son algunas de las claves para lograr metas propuestas por la organización.

Pero el desarrollo y educación de nuestros días requiere una visión moderna acerca de los modelos de aprendizaje. Por esta razón, no podemos centrarnos solo en la aplicación de las teorías tradicionales, sino que debemos evaluar nuevos retos que permitan cumplir los objetivos del proceso de enseñanza y aprendizaje basados en las necesidades actuales de la población.

El aprender investigando es una de las maneras más eficientes de crear el conocimiento. Implica un desarrollo cognitivo alto, en el cual se deban relacionar las estructuras sociales con las necesidades culturales. En este caso, el modelo socio constructivista se convierte 
en una herramienta importante. La investigación científica de la mano de la conservación y del desarrollo social permite crear modelos de conservación basados en las necesidades reales de la sociedad. Si bien es cierto, su aplicación a nivel de educación secundaria es limitada, las investigaciones universitarias pueden profundizar en la problemática social y crear soluciones ecológicamente ambientales que permitan la conservación de los recursos y del desarrollo económico.

Según Altamirano y Salinas (2016), la virtualización acelerada con el inicio del siglo XXI requiere de la aplicación de nuevas tecnologías como parte del quehacer investigativo. El uso de TIC fomenta el interés del estudiantado y promueve la formación de conocimiento a través de las experiencias vividas y la recuperación de los datos almacenados. La ruptura del paradigma de aprender solo en el aula por medio de la repetición es una de las principales herramientas con las que cuenta el personal docente actualmente, con base en un modelo conectivista.

Aunque es claro que el conductismo es la base de muchos procesos de enseñanza y aprendizaje, debemos buscar nuevas metodologías que permitan la integración de estudiantes de la mano de las necesidades actuales. El uso de las TIC, los sistemas de información geográfica (SIG), las redes sociales, entre otros elementos digitales, forman parte de las herramientas que permiten que las nuevas generaciones se interesen en el aprendizaje y, en este caso, la conservación de los recursos naturales; lo cual faculta alcanzar algunos logros como mejorar la comprensión con respecto a la importancia del uso adecuado de los recursos y su regeneración desde los niveles menores de la pirámide educativa costarricense (Cejudo y Almenara, 2015).

Actualmente, existen diversos programas públicos y privados que las escuelas, colegios y universidades pueden adoptar para promover la conservación de recursos naturales, pero el que ha tenido más auge a nivel nacional es el programa bandera azul ecológica. Este impulsa el trabajo en equipo de la mano del socio-constructivismo, como herramienta de mitigación y gestión de impactos negativos en la comunidad escolar. Este tipo de programas fomenta la investigación, ejecución y conciencia socioambiental en el estudiantado en todos los niveles académicos.

Otros programas de promoción ambiental educativa son el reciclaje a nivel de escuela y colegio, la disminución del consumo de agua por medio de llaves y servicios sanitarios especializados, la reutilización 
de material de desecho como llantas en la creación de basureros y juegos para niños, la realización de charlas por medio de personas expertas en conservación ambiental y los talleres que promueven el uso adecuado de los recursos naturales en busca del desarrollo sostenible.

Este tipo de actividades abarca elementos diversos centrados principalmente en el cognitivismo, constructivismo y socio-constructivismo. Deja la puerta abierta para una aplicación más constante del conectivismo al aprovechar las herramientas tecnológicas actuales. Lo que confirma que debemos crear fuentes novedosas de conocimiento para llegar, de manera asertiva, al estudiantado y tomar en cuenta sus necesidades intelectuales para lograr los objetivos propios del proceso de enseñanza y aprendizaje, y de la conservación de los recursos naturales.

\section{Conclusiones}

Las teorías educativas buscan la máxima compresión de la información presente en el proceso de enseñanza y aprendizaje. Es claro cómo cada una se enfoca en diferentes procesos de aprendizaje (desde el punto de vista psicológico), lo que lleva al uso de diversas técnicas didácticas. Por tanto, el estudio y la comprensión de los elementos básicos de cada una de ellas facultan, al personal docente, para el diseño adecuado de recursos que permitan acercarse, de mejor manera, a las metas planteadas y, por ende, al éxito académico esperado.

La educación ambiental debe ser uno de los pilares fundamentales de la educación costarricense desde los primeros años, por lo que el desarrollo de esta debe catalogarse como una necesidad imperante para los sujetos actores sociales del proceso educativo. De esta manera, se inculca la conciencia ambiental desde edades tempranas, por medio de ejercicios conservacionistas investigativos, ferias científicas, actividades prácticas, recursos digitales, entre otros mecanismos que fomentan el desarrollo sostenible y permiten que la ciudadanía del futuro pueda utilizar los recursos naturales de la misma manera que la generación actual.

De la mano con las nociones básicas de las teorías de aprendizaje, los procesos cognitivistas y constructivistas son muy importantes para el desarrollo ambiental en Costa Rica, ya que son las base de muchas de las actividades de esta índole. El crear conocimiento por medio de acciones y resolución de problemas ayuda a interiorizar, de mejor manera, el concepto de conservación biológica y la necesidad de heredar 
un país sostenible en cuanto a sus recursos autóctonos. Si bien es cierto, gran parte de los procesos educativos son repetitivos, verticalistas y memorísticos, los grupos docentes deben afrontar como uno de sus principales retos buscar nuevas maneras de alcanzar los objetivos de aprendizaje, abriendo las puertas a teorías de aprendizaje cada vez más inclusivas y acordes con las poblaciones estudiantiles actuales.

Con base en esta premisa, el desarrollo de nuestro sistema educativo debe dirigir su mirada hacía nuevos modelos que permitan un acercamiento más asertivo y promuevan una educación moderna. De esta manera, la aplicación de teorías como el socio-constructivismo y el conectivismo permite diversificar la educación, no aferrándose a técnicas antiguas en las cuales impera la memoria, la repetición y en donde el profesorado es el amo y señor del conocimiento; sino abordar las necesidades estudiantiles por medio de recursos que sean novedosos e interesantes de manera integral y progresiva.

El uso de la dialéctica, la investigación, los recursos digitales, el trabajo en equipo y la conciencia social como herramientas educativas son algunas de las opciones de mayor importancia que podemos utilizar como motor de cambio, para lograr la conciencia socioambiental necesaria. Los diversos esfuerzos que realizan las universidades y el MEP como el reciclaje, el programa bandera azul, las charlas, las ferias científicas, los programas educativos, las giras y las competencias ambientales forman parte de los ejemplos que evidencian la aplicación de las teorías de aprendizaje como bases epistemológicas para el desarrollo de los recursos didácticos $\mathrm{y}$, por ende, del desarrollo de las habilidades ambientales requeridas en la actualidad.

\section{Referencias}

Alonso, C. (2008). Estilos de aprendizaje. Presente y futuro. Journal of Learning Styles, 1(1). 45-63.

Altamirano, A. y Salinas, Z. (2016). La práctica dicente-investigativa desde la tecnología educativa y el socioconstructivismo [The researching practice of learners based on educational technology and socio-constructivism]. Ciencia Unemi, 9(17), 118-1124.

Aparicio, J., Rodríguez, C., Beltrán, J. y Sampedro, L. (2014). Transversalidad del eje medio ambiente en educación superior. Revista Iberoamericana de Ciencias, 1(1), 163-172. 
Bravo, R. (2005). Aproximación al concepto de transposición didáctica. Revista Folios, 21, 33-45.

Castañon, G. (2017). O cognitivismo é um humanismo. Psicologia Argumento, 25(48), 51-64.

Cejudo, M. y Almenara, J. (2015). Tecnologías de la información y la comunicación (TIC): Escenarios formativos y teorías del aprendizaje. Revista Lasallista de Investigación, 12(2), 25-36.

Chinchilla, J. (2017). Bandera azul ecológica, Costa Rica. Recuperado de http://banderaazulecologica.org/

Craveri, A. M. y Anido, M. (2014). El aprendizaje de matemática como herramienta computacional en el marco de la teoría de los estilos de aprendizaje. Journal of Learning Styles, 2(3), 110-125.

Delgado, J. y Alvarado, M. (2016). Propuesta metodológica de enseñanza y aprendizaje para innovar la educación superior. InterSedes, 17(36), 48-60

Fernández, E. (2016). Aprendizaje constructivista para el análisis de estructuras mediante el uso de un entorno virtual [Constructivist learning for the analysis of structures by using a virtual environment]. Revista Tecnocientifica URU, 9, 41-50.

Francisco, T. y González, J. (2017). Cognición contextualizada: Una propuesta didáctica y psicopedagógica socioconstructivista para la enseñanza-aprendizaje del derecho. Revista Pedagogía Universitaria y Didáctica del Derecho, 4(2), 40-63.

Gutiérrez, L. (2012). Conectivismo como teoría de aprendizaje: Conceptos, ideas y posibles limitaciones. Revista educación y tecnología, 1, 111-122.

Hilario, Y. (2015). Una evaluación epistemológica de la psicología como ciencia. Horizonte de la ciencia, 5(8), 47-54.

Labra, O. (2016). Positivismo y constructivismo: Un análisis para la investigación social. Rumbos TS. Un espacio crítico para la reflexión en Ciencias Sociales, 7, 12-21.

Leiva, C. (2005). Conductismo, cognitivismo y aprendizaje. Revista Tecnología en Marcha, 18(1), 8-17.

Mata, A. (2013). Informe final. Tema: Educación ambiental en Costa Rica. Programa de Mejoramiento del Ambiente Urbano y Conservación del Medio Ambiente Natural. San José: Agencia para la Cooperación Internacional del Japón. Recuperado de http:// 
www.pnuma.org/educamb/reunion_foro_internacional/Informe_Final-Educacion_Ambientalen_Costa_Rica_JICA(3).pdf

Martín, G., Martínez, R., Martín, M., Nieto, M. y Núñez, S. (2017). Acercamiento a las teorías del aprendizaje en la educación superior. UNIANDES EPISTEME, 4(1), 48-60.

Novo, M., y Zaragoza, F. (2006). El desarrollo sostenible: Su dimensión ambiental y educativa. México. Pearson.

Pérez, L., Miguelena, R., y Diallo, A. (2016). La efectividad de la formación en ambientes virtuales de aprendizaje en la educación superior. Campus Virtuales, 5(2), 10-17.

Picazzo, E., Gutiérrez, E., Infante J. y Cantú, P. (2011). La teoría del desarrollo humano y sustentable: Hacia el reforzamiento de la salud como un derecho y libertad universal. Estudios sociales, 19(37), 253-279.

Pozo, J. (2016). La psicología cognitiva y la educación científica. Investigaçoes em ensino de cièncias, 1(2), 110-131.

Rojas, D., Rojas, C. y Fernández, S. (2016). Factores influyentes en motivación y estrategias de aprendizaje en los alumnos de grado. REICE. Revista Iberoamericana sobre Calidad, Eficacia y Cambio en Educación, 14(2), 55-63.

Siemens, G. (2004). Conectivismo: Una teoría de aprendizaje para la era digital. Recuperado de http:/www.fce.ues.edu.sv/uploads/pdf/ siemens-2004-conectivismo.pdf

Sierra, C., Morales, J. y Barrios, R. (2014). Mapas conceptuales como estrategia de enseñanza-aprendizaje en las ciencias ambientales. Itinerario Educativo, 28(64), 163-176.

Sobrino, Á. (2014). Aportaciones del conectivismo como modelo pedagógico post-constructivista. Propuesta educativa, 42, 39-48.

Schunk, D. (1997). Teorías del aprendizaje. México: Pearson Educación.

Torrenteras, J. (2015). Las teorías de aprendizaje y la formación de herramientas técnicas. Revista de Educación a Distancia, 34, 25-31.

Villalobos, E. (2002). Didáctica integrativa y el proceso de aprendizaje. México: Editorial Trillas. 